\title{
Assessment of Health Monitoring Trustworthiness of Avionics Systems
}

\author{
Vladimir Ulansky ${ }^{1}$, Igor Machalin ${ }^{2}$, and Iryna Terentyeva ${ }^{3}$ \\ ${ }^{1}$ Department of Electronics, Robotics, Monitoring and IoT Technologies, National Aviation University, Kyiv, 03058, Ukraine \\ vladimir_ulansky@nau.edu.ua \\ ${ }^{2,3}$ Department of Telecommunication and Radio Electronic Systems, National Aviation University, Kyiv, 03058, Ukraine \\ igor.machalin@nau.edu.ua \\ i_terentyeva@nau.edu.ua
}

\begin{abstract}
The article provides a methodology for assessing the trustworthiness of health monitoring the dismounted avionics systems with automated test equipment (ATE). The indicators include the probabilities of false-positive, falsenegative, true-positive, and true-negative. For the first time, we introduced into consideration the instability of the source of stimulus signal (SSS), the random and systematic component of the measuring channel error, and the reliability characteristics of the systems themselves. We consider a specific case of an exponential distribution of permanent failures and intermittent faults and derive formulas for calculating the trustworthiness indicators. Numerical calculations illustrate how the probabilities of correct and incorrect decisions depend on accuracy parameters. We show that the probabilities of false-positive and false-negative increase much faster than the probabilities of true-positive and true-negative decrease when the standard deviation of stimulus signal increases. For a Very High-Frequency OmniDirectional Range (VOR) receiver, we demonstrate that even with a zero random error generated by the source of the stimulus signal, the probabilities of false-positive and falsenegative are different from zero.
\end{abstract}

\section{INTRODUCTION}

Currently, many airlines and air-force bases worldwide use ATE for monitoring and diagnostics of avionics systems. Examples of such systems are ATEC Series 7 manufactured by Spherea (2017), eCASS - electronic Consolidated Automated Support System made by Lockheed Martin (2020), ATE IRIS 2000/IRS 1200 system manufactured by

\footnotetext{
Vladimir Ulansky et al. This is an open-access article distributed under the terms of the Creative Commons Attribution 3.0 United States License, which permits unrestricted use, distribution, and reproduction in any medium, provided the original author and source are credited.

https://doi.org/10.36001/IJPHM.2021.v12i2.2907
}

Aeroflex (2005), and many others. The main goal of using ATE is to reduce maintenance costs over the lifetime of avionics, which depends on solving the following major tasks: increasing ATE versatility (an increase in the number of types of tested avionics systems) and improves the validity of operability checking and troubleshooting. For instance, the eCASS is compatible with more than 550 sets of test programs that test avionics systems on multiple platforms. Modern ATE are built on a modular principle using interface PXI bus and standard digital interface for programmable instrumentation IEC-60488-1 (2004) and IEC-60488-2 (2004). There are two primary directions of ATE development: generality and openness (Ma et al., 2013); the main aspects of these directions investigated by (Droste \& Guilbeaux, 2009), (Evlanov, 1979), and (Stora \& Droste, 2003).

At the stages of the design and operation of ATE for avionics systems, the task of assessing the trustworthiness indicators of health monitoring is highly topical. Indeed, inspection errors such as false-positive and false-negative can lead to economic losses and flight safety reduction.

Let us look at how false-positive and false-negative events affect the cost of avionics maintenance and flight safety. Modern digital avionics systems present modular units with high requirements for testability and maintainability (eCASS, 2020). An avionics system usually consists of one or several line-replaceable units (LRU) or line-replaceable modules (LRM). Each LRU or LRM comprises a set of shop replaceable units (SRU) representing a printed circuit board assemblies (PCB). Based on the three-component level of avionics systems (LRU, SRU, nonrepairable element), the following three levels of maintenance are composed: organizational maintenance (O-level), intermediate maintenance (I-level), and depot maintenance (D-level). The O-level maintenance targets the isolation of defected LRU at the aircraft parking. I-level maintenance performs isolation 
of the LRU failure with depth to SRU. At I-level, specialized ATE automates most of the test procedures. D-level maintenance performs isolation of failure with depth to nonrepairable elements. The specialized repair centers or original equipment manufacturers typically conduct D-level maintenance. As has been shown (Bao et al., 2006; Raza et al., 2017; Raza \& Ulansky, 2018; Ross, 2003), the maintenance system may comprise all three levels or consist of the two if the only O- and I-levels or O- and D-levels are applicable.

As is well known (Raza, 2018; Raza \& Ulansky, 2020), any two or three-level maintenance options must check the operability of the dismantled LRU or LRM. We illustrate this statement by the diagram presented in Figure 1 for two-level maintenance, including O- and I-level. As we can see in Figure 1, the dismounted LRU can be judged as operable or inoperable by the results of testing with the help of ATE. Since the flow of dismounted LRU includes both inoperable and operable units, one of four incompatible events may occur according to the checking results: true-positive, falsepositive, true-negative, or false-negative. When a truepositive or false-negative event occurs, the unit is shipped to the warehouse of spare LRU. On the other hand, when a truenegative or false-positive event occurs, the unit is delivered to the manufacturer or outsourcing company for repair.

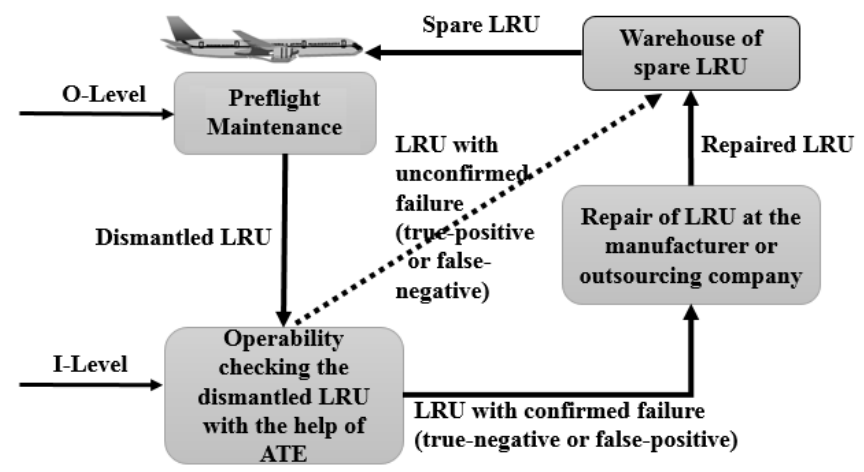

Figure 1. Diagram of an LRU circulation for a two-level maintenance option comprising $\mathrm{O}$ - and I-level

Thus, from the diagram in Figure 1, the occurrence of a falsenegative (undetected failure) when checking the LRU will ultimately lead to installing a faulty unit onboard the aircraft that may have an impact on flight safety. An example of such an onboard safety-related avionics system is the Instrument Landing System (ILS), which usually comprises three and sometimes even more identical receivers with a k-out-of-n reliability structure (Rausand \& Hoyland, 2003). However, the appearance of a false-positive event will lead to shipping the operable LRU to the manufacturer or outsourcing company for repair, which we associate with economic losses.
Let us consider the most significant publications in this area. (Breitgand et al., 2011) developed a specific algorithm for controlling the rate of false-positive and false-negative. (Ho et al., 2012) proposed a false-positive and false-negative assessment mechanism that collects corresponding errors from real-world traffic and statistically analyzes these cases. (Mane et al., 2004) considered a capture-recapture-based method to estimate false-negatives when using two or more independent classifiers. (Foss \& Zaiane, 2008) proposed an algorithm for computation true-positive and false-positive rates using a statistical error rate algorithm. (Ebrahimi, 2008) considered the problem of determining thresholds controlling both false-positives and false-negatives by using a specific risk function. (Scott, 2007) proposed performance measures to evaluate and compare classifiers concerning minimizing the probability of false-negative whereas restricting the probability of a false positive. (Evlanov, 1979; Kudritsky et al., 1977; Ulansky, 1992) considered analytical methods for calculating the probabilities of false-positive and falsenegative. The measurement result includes the actual value of the monitoring parameter and additive random noise in these studies.

Note that all the cited studies do not consider the specifics of health monitoring the avionics systems. We should also note that the metric F1 score, widely used in binary classification and statistical analysis (Chen, 2019; Hossin \& Sulaiman, 2015; Manning et al., 2008; Sokolova et al., 2006), is impractical to use for assessing the trustworthiness of monitoring avionics systems for two reasons. Firstly, it gives equal importance to precision and recall, but in practice, different types of classification errors lead to various losses (Hand \& Christen, 2018). Secondly, there is only a statistical formula for calculating the F1 score. Hence, to assess the increase or decrease in trustworthiness when changing the testing procedure is possible only through numerous tests, which leads to high costs.

Therefore, this study provides a methodology for calculating the trustworthiness indicators of the health monitoring of avionics systems on the example of navigation and landing systems; we consider the instability of the SSS, the accuracy characteristics, and the reliability of the systems themselves. We examine the primary sources of measurement errors in detail when testing VOR receivers and formulate the events that lead to the correct and incorrect decisions. We derive the generalized expressions of trustworthiness indicators and specific formulas for the case of an exponential distribution of permanent failures and intermittent faults. Next, we illustrate the dependence of the probabilities of correct and incorrect decisions when testing VOR receivers versus different accuracy parameters. Finally, we consider an example of assessing the trustworthiness indicators when testing ILS with ATE. 


\section{Trustworthiness AsSESSMent Methodology}

The following section outlines the general methodology of assessing the health monitoring trustworthiness of avionics systems on the example of a navigation system.

\subsection{Block Diagram of VOR Receiver Health Monitoring}

Let us determine the trustworthiness indicators of the health monitoring of the onboard VOR radio receiver, which represents a typical avionics LRU. Onboard equipment VOR provides aircraft navigation on land lighthouses VOR. This equipment allows to solve the following navigation tasks:

- Determine the magnetic bearing of the VOR ground-based radio beacon;

- Determine the location of the aircraft using the magnetic bearings of two VOR radio beacons;

- Determine the drift angle in flight.

According to Aeronautical Information Manual (2017), an error of $\pm 1^{\circ}$ usually characterizes the accuracy of determining VOR radio beacons' bearings using onboard equipment. When checking the VOR receiver's operability with the help of ATE, the tested parameter is the error of azimuth measurement.

Figure 2 shows a block diagram of monitoring the health of an airborne VOR receiver. A specific stimulus signal of magnitude $A$ must be applied to the VOR receiver to monitor azimuth measurement error. Therefore, the controller applies a control signal to the SSS, which, acting on the corresponding controls, sets the required shape and magnitude of the stimulus signal at its output.

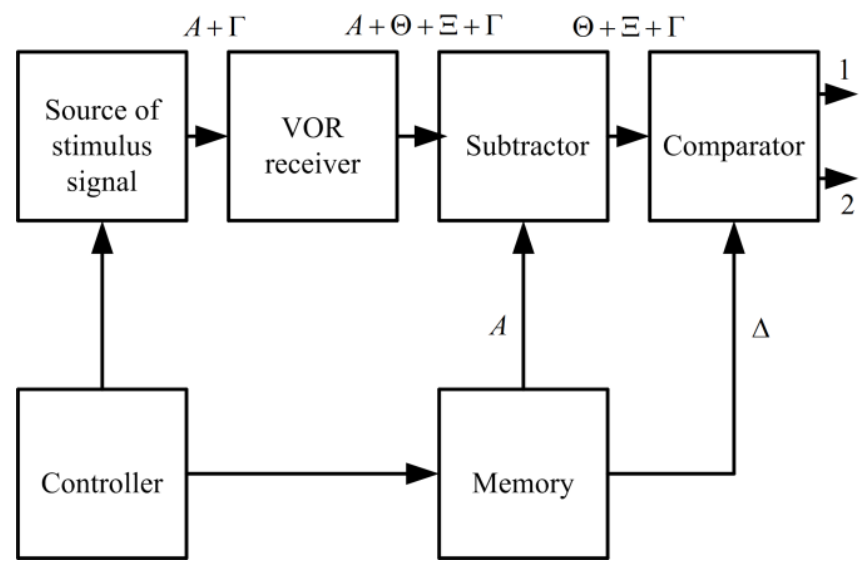

Figure 2. Block diagram of VOR receiver health monitoring

Since the SSS has finite stability, instead of the required value of the stimulus signal $A$, it applies a signal of magnitude $A+$ $\Gamma$ to the input of the VOR receiver, where $\Gamma$ is the random component of the SSS error. Further, we assume that the systematic component of the SSS error was eliminated as a result of planned calibrations; therefore, we do not consider it. In turn, the VOR receiver introduces the error $\Theta+\Xi$ into the signal $A$ applied to its input, where $\Theta$ and $\Xi$ are, respectively, the systematic and random components of the error of azimuth measurement. Then, by the signal from the controller, the memory feeds the value of signal $A$ to the subtractor. Thus, we can represent the difference signal at the output of the subtractor as follows:

$$
Y=\Theta+\Xi+\Gamma
$$

Signal $Y$ feeds into the comparator that uses the following decision rule. If $|y| \leq|\Delta|$, then it makes the decision "the error of the azimuth measurement in the tolerance" (comparator output 1 in Figure 2), where $\Delta$ is the limit of the permissible error of azimuth measurement by the VOR receiver and $y$ is the realization of the random variable $Y$. If $|y|>|\Delta|$, then it makes the decision "the error of the azimuth measurement is out of the tolerance" (comparator output 2 in Figure 2). Since both the subtractor and comparator are microprocessor devices, we can neglect the errors of the subtraction and comparison operations.

The random errors in Equation (1) are not correlated with each other. Therefore, between the random variables $\Gamma, \Theta$, and $\Xi$, there is an additive relationship. Indeed, the random error $\Gamma$ is not dependent on $\Theta$ and $\Xi$ because it is generated by the external source of stimulus signal. Further, for a specific VOR receiver, the systematic measurement error $\Theta$ is not a random variable, but a constant value, which depends on the accuracy of the initial setup of the measuring path of the VOR receiver at the manufacturer. The measuring channel of the VOR receiver receives, filters, amplifies and demodulates high-frequency signals from the antenna, as well as converts analog low-frequency signals into a digital code, which is transmitted to the onboard computer. In the process of converting a high-frequency signal into a digital code, a significant number of different operations (analog and digital) are performed, which cause the appearance of a random component of the azimuth measurement error $\Xi$. The random component $\Xi$ fluctuates around some constant value, which is the systematic component of the azimuth measurement error. Moreover, for different VOR receivers with varying values of the systematic component, the standard deviations of these fluctuations are practically the same. Due to these reasons, it is assumed that there is an additive relationship between the random variables $\Theta$ and $\Xi$.

\subsection{Formalization of Incompatible Events Based on Decision-making when Monitoring the Health of VOR Receiver}

Due to the presence of a random component $Z=\Xi+\Gamma$ in the difference signal $Y$, the decisions made by the comparator may turn out to be erroneous. In this case, incorrect decisions such as false-positive $\left(S_{F P}\right)$ and false-negative $\left(S_{F N}\right)$ take place accordingly when the following complex events occur: 


$$
\begin{aligned}
& S_{F P}=\{|\Theta| \leq|\Delta| \cap|\Theta+Z|>|\Delta|\} \\
& S_{F N}=\{|\Theta|>|\Delta| \cap|\Theta+Z| \leq|\Delta|\}
\end{aligned}
$$

where $\cap$ is the symbol of the intersection of different events.

Event (2) means that the systematic error of azimuth measurement is within the tolerance, but its measured value is out of tolerance. Event (3) implies that the systematic error of azimuth measurement is out of tolerance, but its measured value is within the tolerance.

On the other hand, the comparator can make the correct decisions, such as true-positive $\left(S_{T P}\right)$ and true-negative $\left(S_{T N}\right)$.

$$
\begin{aligned}
& S_{T P}=\{|\Theta| \leq|\Delta| \cap|\Theta+Z| \leq|\Delta|\} \\
& S_{T N}=\{|\Theta|>|\Delta| \cap|\Theta+Z|>|\Delta|\}
\end{aligned}
$$

Event (4) means that the systematic error of azimuth measurement and its measured value is within the tolerance. Event (5) implies that the systematic error of azimuth measurement and its measured value is out of tolerance.

As we can see from relations (2)-(5), events $S_{F P}, S_{F N}, S_{T P}$, and $S_{T N}$ represent a group of mutually exclusive events; the sum of their probabilities is unity.

\subsection{Determination of Probabilities of Correct and Incorrect Decisions}

Figure 3 shows the graph of decision-making when checking the VOR receiver, where $P$ is the a priori probability of the VOR receiver operability and $q(\theta)$ is the probability density function (PDF) of the systematic component of the azimuth measurement error over the set of the same VOR receivers.

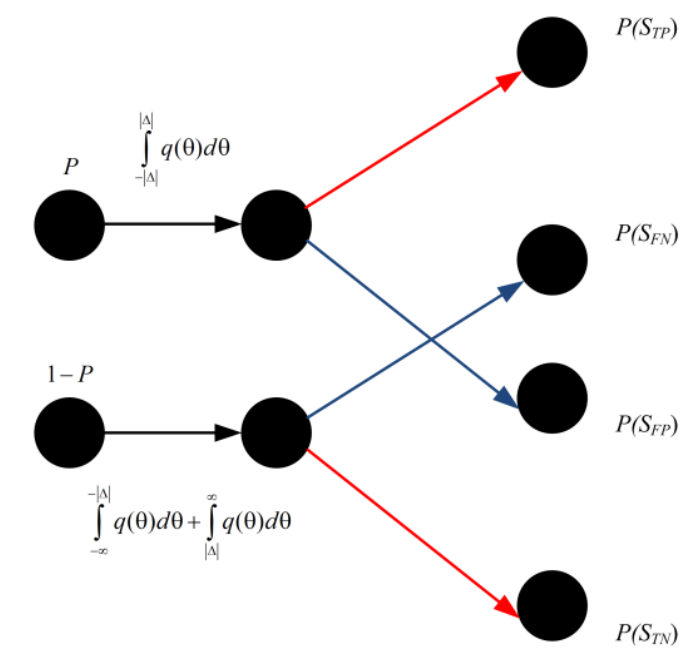

Figure 3. The graph of decision-making when checking the VOR receiver operability

According to the graph of decision-making in Figure 3, we can write the following obvious equations:

$$
\begin{gathered}
P=P\left(S_{F P}\right)+P\left(S_{T P}\right) \\
1-P=P\left(S_{F N}\right)+P\left(S_{T N}\right)
\end{gathered}
$$

We show the expressions for the prior probabilities $P$ and $1-$ $P$ on the edges of the decision-making graph in Figure 3.

Since the random variable $\mathrm{Z}$ is the sum of two independent continuous random variables $\Xi$ and $\Gamma$, its PDF $g(z)$ can be represented as a composition of the PDF of these random variables, i.e.,

$$
g(z)=\int_{-\infty}^{\infty} f(z-\gamma) \varphi(\gamma) d \gamma
$$

where $f(\xi)$ and $\varphi(\gamma)$ are PDF of random variables $\Xi$ and $\Gamma$, respectively.

Using the theorems of addition and multiplication of probabilities and considering Equation (8), we determine the probabilities of events (2)-(5) as follows:

- the probability of false-positive

$$
\begin{aligned}
& P\left(S_{F P}\right)=P-P\left(S_{T P}\right)=\int_{-|\Delta|}^{|\Delta|} q(\theta) d \theta- \\
& \int_{-|\Delta|}^{|\Delta|} q(\theta) \int_{-|\Delta|-\theta}^{|\Delta|-\theta} \int_{-\infty}^{\infty} f(z-\gamma) \varphi(\gamma) d \gamma d z d \theta
\end{aligned}
$$

- the probability of false-negative

$$
\begin{aligned}
& P\left(S_{F N}\right)=\int_{-\infty}^{-|\Delta|} q(\theta) \int_{-|\Delta|-\theta}^{|\Delta|-\theta} \int_{-\infty}^{\infty} f(z-\gamma) \varphi(\gamma) d \gamma d z d \theta+ \\
& \int_{|\Delta|}^{\infty} q(\theta) \int_{-|\Delta|-\theta}^{|\Delta|-\theta} \int_{-\infty}^{\infty} f(z-\gamma) \varphi(\gamma) d \gamma d z d \theta
\end{aligned}
$$

- the probability of true-positive

$$
P\left(S_{T P}\right)=\int_{-|\Delta|}^{|\Delta|} q(\theta) \int_{-|\Delta|-\theta}^{|\Delta|-\theta} \int_{-\infty}^{\infty} f(z-\gamma) \varphi(\gamma) d \gamma d z d \theta
$$

- the probability of true-negative

$$
P\left(S_{T N}\right)=\int_{-\infty}^{-|\Delta|} q(\theta) \int_{-\infty}^{-|\Delta|-\theta} \int_{-\infty}^{\infty} f(z-\gamma) \varphi(\gamma) d \gamma d z d \theta+
$$

$$
\int_{|\Delta|}^{\infty} q(\theta) \int_{|\Delta|-\theta}^{\infty} \int_{-\infty}^{\infty} f(z-\gamma) \varphi(\gamma) d \gamma d z d \theta
$$




\subsection{Case of Sudden Failures}

ATE checks the health of VOR units rejected during operation by built-in test equipment (BITE) and dismounted from the boards of the aircraft fleet. In the flow of dismounted units, we can observe VOR receivers with permanent failures and receivers removed from the aircraft boards due to intermittent faults registered by BITE. Indeed, according to (Ilarslan \& Ungar, 2016; Khan et al., 2014), the rate of intermittent faults for avionics is from $20 \%$ to $50 \%$ of the total percentage of removals. Due to the presence in the flow of dismounted units, both operable and failed (i.e., with permanent failures) VOR receivers, all the formulated events (2)-(5) are possible.

At the initial stage of equipment operation, the laws of degradation of monitoring parameters are generally unknown. Therefore, the calculation of reliability is carried out for the exponential distribution law of permanent failures. So, further, we will assume that only sudden failures occur in VOR receivers.

The systematic component of the azimuth measurement error depends on the accuracy of the initial setup of the measuring path of the VOR receiver and the presence of defects in it. By defects, we mean breaks, short circuits in the electrical circuitry of the dismounted VOR receiver, and other quantitative changes in the properties of the components, leading to a sudden exit of the systematic part of the azimuth measurement error beyond tolerance.

Thus, we can present the systematic error of azimuth measurement as follows:

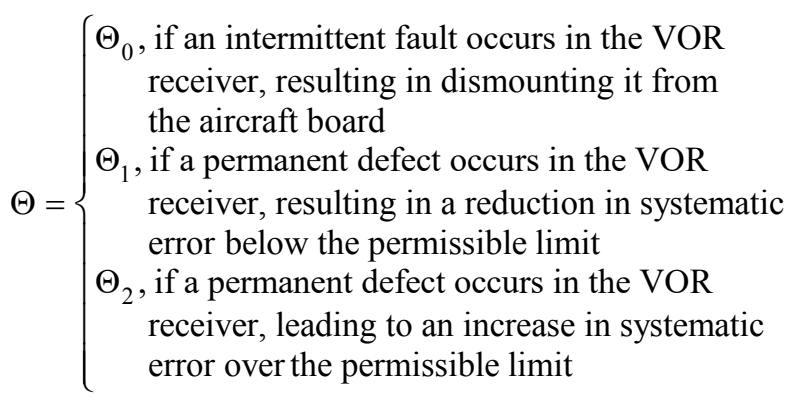

The following apparent conditions are met: $\left|\Theta_{0}\right| \leq|\Delta|,\left|\Theta_{1}\right|>$ $|\Delta|$, and $\left|\Theta_{2}\right|>|\Delta|$. These conditions mean that in the absence of permanent failure in the VOR receiver, all possible values of the systematic error lie within tolerance. In the presence of permanent failure, the value of the systematic component is out of tolerance.

Figure 4 shows the graph of transitions of the VOR receiver to states leading to dismounting from the aircraft. In Figure $4, \lambda_{0}$ is the rate of intermittent faults transferring the VOR receiver to the state $\mathrm{D}_{0}$, in which it is dismounted from the aircraft board not having any permanent failure, $\lambda_{1}$ and $\lambda_{2}$ are the rates of permanent failures that transfer the VOR receiver from the operable state to inoperable states $\mathrm{D}_{1}$ and $\mathrm{D}_{2}$, corresponding to a decrease and increase in systematic error by an amount higher than permissible.

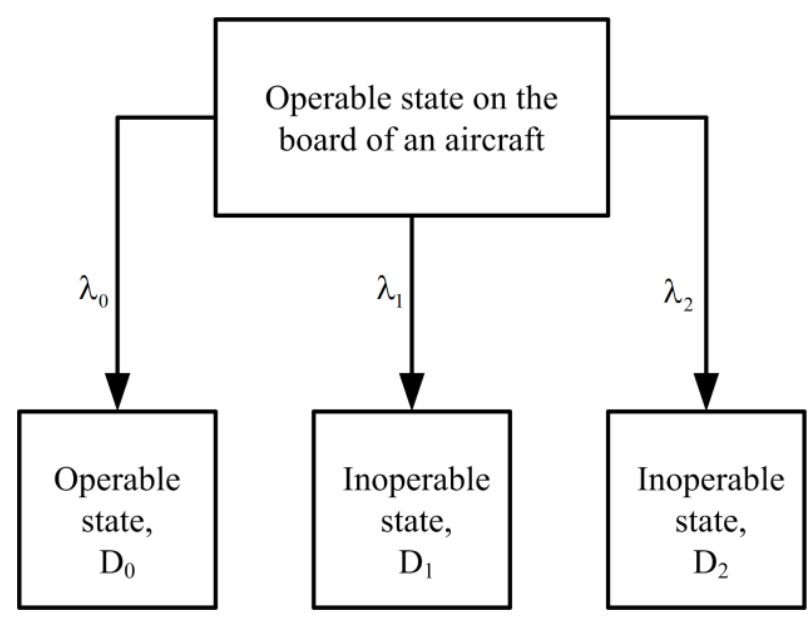

Figure 4. The graph of transitions of VOR receiver from the board of an aircraft

Thus, the VOR receiver that does not have a permanent failure can be dismounted from an aircraft due to one or more intermittent faults recorded by the BITE during flight (state $\mathrm{D}_{0}$ ). According to Figure 1, ATE will test the dismounted unit at the I-level maintenance and with probability $P\left(S_{T P}\right)$ does not confirm the existence of a permanent failure. Moreover, conventional ATE will not also detect the root of intermittent faults (Anderson, 2014).

Inoperable states $\mathrm{D}_{1}$ and $\mathrm{D}_{2}$ correspond to situations when a permanent failure occurs during flight and BITE detects it.

As we can see in Figure 4, at the exponential failure distribution, the total transition rate is

$$
\Lambda=\lambda_{0}+\lambda_{1}+\lambda_{2}
$$

Based on the above reasoning, we can present the PDF of the systematic error in measuring the azimuth of the VOR receivers coming to the health monitoring as follows:

$$
q(\theta)=\frac{\lambda_{0}}{\Lambda} q_{0}(\theta)+\frac{\lambda_{1}}{\Lambda} q_{1}(\theta)+\frac{\lambda_{2}}{\Lambda} q_{2}(\theta)
$$

where $q_{0}(\theta), q_{1}(\theta)$, and $q_{2}(\theta)$ are, respectively, the PDF of the systematic component of the azimuth measurement error in the absence and presence of permanent failures.

Considering Equation (14) and the fact that random variables $\Theta_{0}, \Theta_{1}$, and $\Theta_{2}$ have non-overlapping intervals of existence, we transform the probabilities (9)-(12) to the following form:

- the probability of false-positive 


$$
P\left(S_{F P}\right)=\frac{\lambda_{0}}{\Lambda}\left[\begin{array}{l}
\int_{-|\Delta|}^{|\Delta|} q_{0}(\theta) d \theta- \\
\int_{-|\Delta|}^{|\Delta|} q_{0}(\theta) \int_{-|\Delta|-\theta-\infty}^{|\Delta|-\theta} \int_{-\infty}^{\infty} f(z-\gamma) \varphi(\gamma) d \gamma d z d \theta
\end{array}\right]
$$

- the probability of false-negative

$$
\begin{aligned}
& P\left(S_{F N}\right)=\frac{\lambda_{1}}{\Lambda}\left[\int_{-\infty}^{-|\Delta|} q_{1}(\theta) \int_{-|\Delta|-\theta-\infty}^{|\Delta|-\theta} \int_{-\infty}^{\infty} f(z-\gamma) \varphi(\gamma) d \gamma d z d \theta\right]+ \\
& \frac{\lambda_{2}}{\Lambda}\left[\int_{|\Delta|}^{\infty} q_{2}(\theta) \int_{-|\Delta|-\theta \mid-\infty}^{|\Delta|-\theta} \int_{-\infty}^{\infty} f(z-\gamma) \varphi(\gamma) d \gamma d z d \theta\right]
\end{aligned}
$$

- the probability of true-positive

$$
P\left(S_{T P}\right)=\frac{\lambda_{0}}{\Lambda}\left[\int_{-|\Delta|}^{|\Delta|} q_{0}(\theta) \int_{-|\Delta|-\theta}^{|\Delta|-\theta} \int_{-\infty}^{\infty} f(z-\gamma) \varphi(\gamma) d \gamma d z d \theta\right]
$$

- the probability of true-negative

$$
\begin{aligned}
& P\left(S_{T N}\right)=\frac{\lambda_{1}}{\Lambda}\left[\int_{-\infty}^{-|\Delta|} q_{1}(\theta) \int_{-\infty}^{-|\Delta|-\theta} \int_{-\infty}^{\infty} f(z-\gamma) \varphi(\gamma) d \gamma d z d \theta\right]+ \\
& \frac{\lambda_{2}}{\Lambda}\left[\int_{|\Delta|}^{\infty} q_{2}(\theta) \int_{|\Delta|-\theta-\infty}^{\infty} \int_{-\infty}^{\infty} f(z-\gamma) \varphi(\gamma) d \gamma d z d \theta\right]
\end{aligned}
$$

We can assume that the random variable $\Theta_{0}$ has a normal PDF since the distribution of $\Theta_{0}$ depends on the accuracy of tuning the channel for measuring the azimuth of the VOR receiver at the manufacturer. When setting up the measuring channels of electronic devices, the rule of three sigmas usually has a place. Since $\left|\Theta_{0}\right| \leq|\Delta|$, it is evident that the random variable $\Theta_{0}$ has a truncated normal distribution with the mean square deviation of $\sigma_{\theta}=|\Delta| / 3$ and mathematical expectation $E\left(\Theta_{0}\right)=$ 0 .

In the design and early stages of VOR receives' operation, engineers do not know the mathematical expectation and the standard deviation of the systematic component of the error in measuring the azimuth by the failed VOR receivers. However, it is usually possible to determine the boundary values of this error from the operational algorithm of the VOR receiver. A uniform distribution has the maximum entropy with the known boundaries of the systematic measurement error (Lisman \& van Zuylen, 1972). Therefore, we assume that the random variables $\Theta_{1}$ and $\Theta_{2}$ have a uniform distribution.
Substituting into Equation (14) the values of the PDF of random variables $\Theta_{0}, \Theta_{1}$, and $\Theta_{2}$, we obtain

$$
q(\theta)=\frac{c \lambda_{0} \exp \left(-\theta^{2} / 2 \sigma_{\theta}^{2}\right)}{\sqrt{2 \pi} \sigma_{\theta} \Lambda}+\frac{\lambda_{1}}{\left(-\Delta_{l}-|\Delta|\right) \Lambda}+\frac{\lambda_{2}}{\left(\Delta_{h}-|\Delta|\right) \Lambda}
$$

where $\Delta_{l}$ and $\Delta_{h}$ are, respectively, the lower and upper boundaries of the systematic component of the error in azimuth measurement in the presence of defects in the VOR receiver, and $c$ is the normalization constant.

$$
c=\left[\frac{1}{\sqrt{2 \pi} \sigma_{\theta}} \int_{-|\Delta|}^{|\Delta|} \exp \left(-\theta^{2} / 2 \sigma_{\theta}^{2}\right) d \theta\right]^{-1}
$$

Substituting Equations (19) and (20), and the PDF of the random errors $\Xi$ and $\Gamma$ into Equations (15)-(18) after corresponding transformations we obtain the following formulas:

$$
\begin{aligned}
& P\left(S_{F P}\right)=\frac{\lambda_{0}}{\Lambda \sqrt{2 \pi}}\left[\int_{\frac{-|\Delta|}{\sigma_{\theta}}}^{\frac{|\Delta|}{\sigma_{\theta}}} \exp \left(-x^{2} / 2\right) d x-\frac{1}{\sqrt{2 \pi}} \times\right. \\
& \left.\frac{-|\Delta|}{\sigma_{\theta}} \exp \left(-x^{2} / 2\right) \int_{\frac{-|\Delta|-x \sigma_{\theta}}{\sqrt{\sigma_{\xi}^{2}+\sigma_{\gamma}^{2}}}}^{\frac{|\Delta|-x \sigma_{\theta}}{\sqrt{\sigma_{\xi}^{2}+\sigma_{\gamma}^{2}}}} \exp \left(-u^{2} / 2\right) d u d x\right] \times
\end{aligned}
$$

$$
\left[\frac{1}{\sqrt{2 \pi}} \int_{\frac{-|\Delta|}{\sigma_{\theta}}}^{\frac{|\Delta|}{\sigma_{\theta}}} \exp \left(-x^{2} / 2\right) d x\right]^{-1}
$$

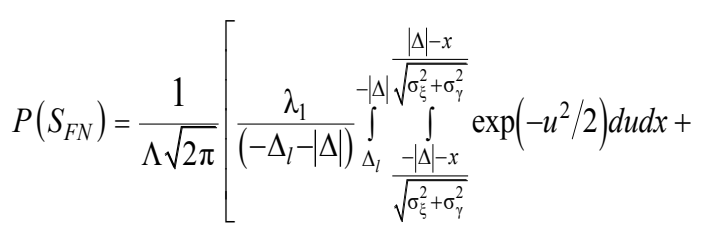

$$
\left.\frac{\lambda_{2}}{\left(\Delta_{h}-|\Delta|\right)} \int_{|\Delta|}^{\Delta_{h}} \frac{\frac{|\Delta|-x}{\sqrt{\sigma_{\xi}^{2}+\sigma_{\gamma}^{2}}}}{\sqrt{\sqrt{\sigma_{\xi}^{2}+\sigma_{\gamma}^{2}}}} \exp \left(-u^{2} / 2\right) d u d x\right]
$$




$$
\begin{aligned}
& P\left(S_{T P}\right)=\frac{\lambda_{0}}{2 \pi \Lambda}\left[\frac{-|\Delta|}{\sigma_{\theta}} \frac{|\Delta|}{\sigma_{\theta}} \exp \left(-\frac{x^{2}}{2} \int_{\frac{-|\Delta|-x \sigma_{\theta}}{\sqrt{\sigma_{\xi}^{2}+\sigma_{\gamma}^{2}}}}^{\frac{|\Delta|-x \sigma_{\theta}}{\sqrt{\sigma_{\xi}^{2}+\sigma_{\gamma}^{2}}}} \exp \left(-\frac{u^{2}}{2}\right) d u d x\right] \times\right. \\
& {\left[\frac{1}{\sqrt{2 \pi}} \int_{\frac{-|\Delta|}{\sigma_{\theta}}}^{\frac{|\Delta|}{\sigma_{\theta}}} \exp \left(-x^{2} / 2\right) d x\right]^{-1}} \\
& P\left(S_{T N}\right)=\frac{1}{\Lambda \sqrt{2 \pi}}\left[\frac{\lambda_{1}}{\left(-\Delta_{l}-|\Delta|\right)} \int_{\Delta_{l}}^{-|\Delta|} \int_{-\infty}^{\frac{-|\Delta|-x}{\sqrt{\sigma_{\xi}^{2}+\sigma_{\gamma}^{2}}}} \exp \left(-\frac{u^{2}}{2}\right) d u d x+\right. \\
& \left.\frac{\lambda_{2}}{\left(\Delta_{h}-|\Delta|\right)} \int_{|\Delta|}^{\Delta_{h}} \frac{|\Delta|-x}{\sqrt{\sigma_{\xi}^{2}+\sigma_{\gamma}^{2}}} \int^{\infty} \exp \left(-\frac{u^{2}}{2}\right) d u d x\right]
\end{aligned}
$$

\section{RESULTS AND DISCUSSION}

Example 1. Let us consider an example of calculating the trustworthiness indicators when monitoring the health of dismounted VOR receivers. The tested VOR equipment operates in the frequency range $108.0-117.975 \mathrm{MHz}$. There are 160 fixed frequencies (channels) for work with VOR beacons. Azimuth measurement error is no more than $0.5^{\circ}$. We use the following initial data: $|\Delta|=0.00873 \mathrm{rad}, \Delta_{h}=-\Delta_{l}$ $=0.04365 \mathrm{rad}, \sigma_{\xi}=\sigma_{\theta}=0.00291 \mathrm{rad}$, and $\sigma_{\gamma}=0.00175 \mathrm{rad}$.

Table 1 illustrates the dependence of trustworthiness indicators on the fraction of VOR receivers with intermittent faults arriving for health monitoring using ATE when $\lambda_{1} / \Lambda=$ $\lambda_{2} / \Lambda=\left(1-\lambda_{0} / \Lambda\right) / 2$. The fraction of VOR receivers dismounted from the aircraft due to intermittent faults $\left(\lambda_{0} / \Lambda\right)$ corresponds to the operable units that do not have permanent failures.

As we can see in Table 1, the probability of false-positive increases, and the probability of false-negative decreases with an increase in the fraction of operable VOR receivers. At the same time, the probability of true-positive increases, and true-negative decreases.

To explain this behavior of trustworthiness indicators, we should consider their dependence on the ratio $\lambda_{0} / \Lambda$ determined by the following equations:
Table 1. Trustworthiness indicators versus the fraction of VOR receivers that do not have permanent failures

\begin{tabular}{l|c|c|c|c}
\hline \multirow{2}{*}{$\begin{array}{l}\text { Trustworthiness } \\
\text { indicator }\end{array}$} & \multicolumn{4}{|c}{$\begin{array}{l}\text { The fraction of operable VOR } \\
\text { receivers, } \lambda_{0} / \Lambda\end{array}$} \\
\cline { 2 - 5 } & 0.2 & 0.4 & 0.6 & 0.8 \\
\hline $\begin{array}{l}\text { Probability of } \\
\text { false-positive }\end{array}$ & 0.010 & 0.020 & 0.030 & 0.040 \\
\hline $\begin{array}{l}\text { Probability of } \\
\text { false-negative }\end{array}$ & 0.031 & 0.023 & 0.016 & 0.008 \\
\hline $\begin{array}{l}\text { Probability of } \\
\text { true-positive }\end{array}$ & 0.190 & 0.380 & 0.570 & 0.760 \\
\hline $\begin{array}{l}\text { Probability of } \\
\text { true-negative }\end{array}$ & 0.769 & 0.577 & 0.384 & 0.192 \\
\hline
\end{tabular}

As we can see in Equations (25) and (26), with an increase in the ratio $\lambda_{0} / \Lambda$, the sum of the probabilities of false-positive and true-positive increases and the sum of the probabilities of false-negative and true-negative decreases. Since we did not change the accuracy characteristics of the measuring channel when making calculations, the dependencies in Table 1 are linear.

Let us investigate the dependence of the trustworthiness indicators of health monitoring on the accuracy characteristics of the measuring channel when $\lambda_{0} / \Lambda=0.4$ and $\lambda_{1} / \Lambda=\lambda_{2} / \Lambda=0.3$.

Figures 5 and 6 show the dependence of the probabilities of false-positive and true-positive versus standard deviation of the systematic component of the azimuth measurement error.

From Figures 5 and 6, we can see that the probability of falsepositive increases from $0.4 \%$ to $5.4 \%$, and the probability of true-positive decreases from $39.5 \%$ to $34.5 \%$ with increasing the standard deviation of the systematic component of the azimuth measurement error in the interval $(0,0.01) \mathrm{rad}$. Thus, in the interval of $\sigma_{\theta}$ variation, the probability of a false-positive increases by $1250 \%$, and the probability of a true-positive decreases only by $12.7 \%$. 


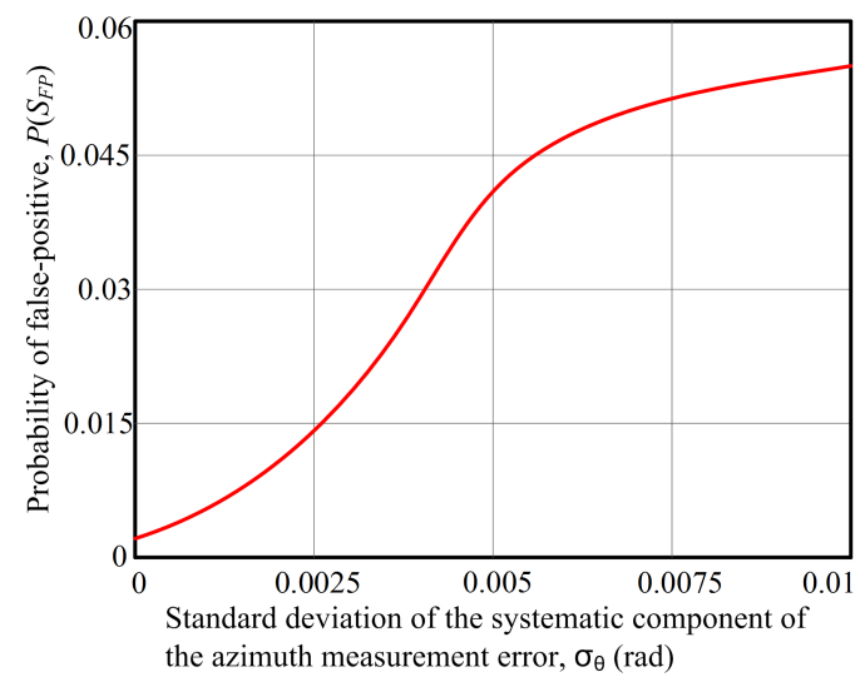

Figure 5. Dependence of the probability of false-positive versus standard deviation of the systematic component of the azimuth measurement error

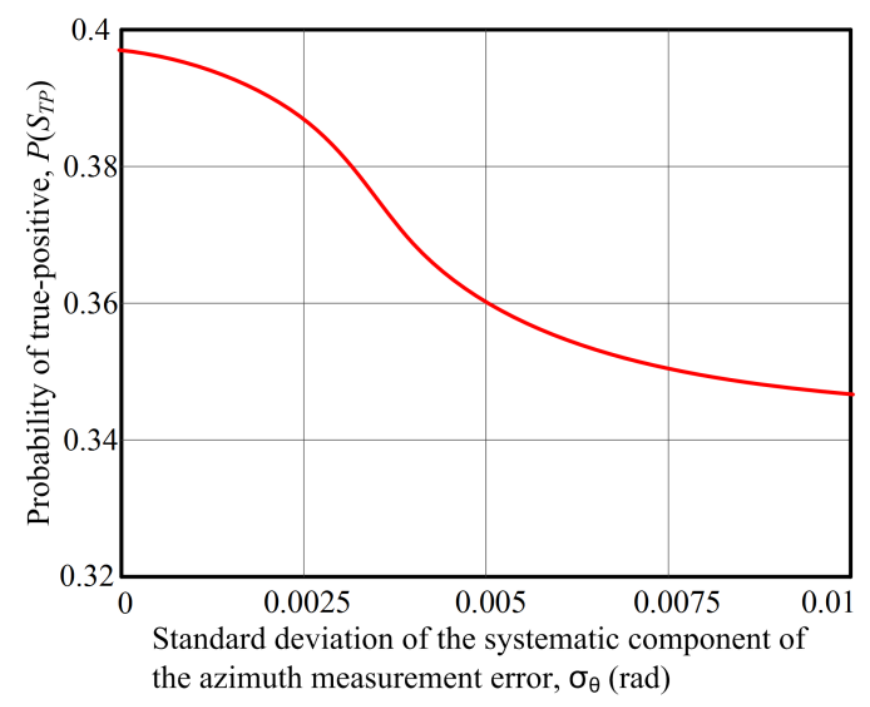

Figure 6. Dependence of the probability of true-positive versus standard deviation of the systematic component of the azimuth measurement error

Figures 7 and 8 show the dependence of the probabilities of false-positive and true-positive versus standard deviation of the random error generated by the SSS.

Analyzing Figures 7 and 8, we can make the following conclusions. The probability of false-positive is very much dependent on the standard deviation of the random error generated by the SSS. Indeed, when $\sigma_{\gamma}$ changes from 0 to 0.01 rad, the probability $P\left(S_{F P}\right)$ increases from $2 \%$ to $17 \%$.

The probability of true-positive decreases when the standard deviation of the random error generated by the SSS increases. When $\sigma_{\gamma}$ changes from 0 to $0.01 \mathrm{rad}$, the probability $P\left(S_{T P}\right)$ decreases from $38 \%$ to $23 \%$.

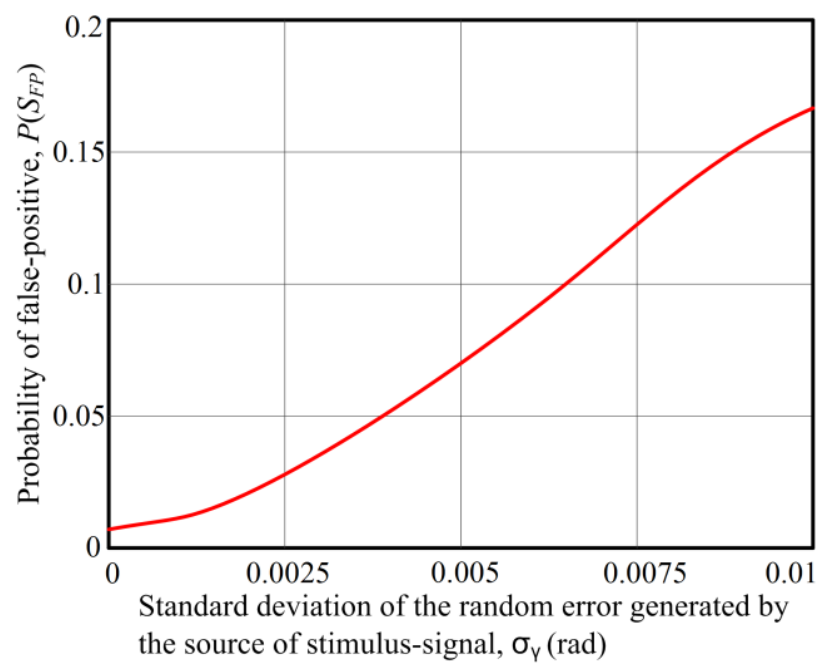

Figure 7. Dependence of the probability of false-positive versus standard deviation of the random error generated by the source of the stimulus signal

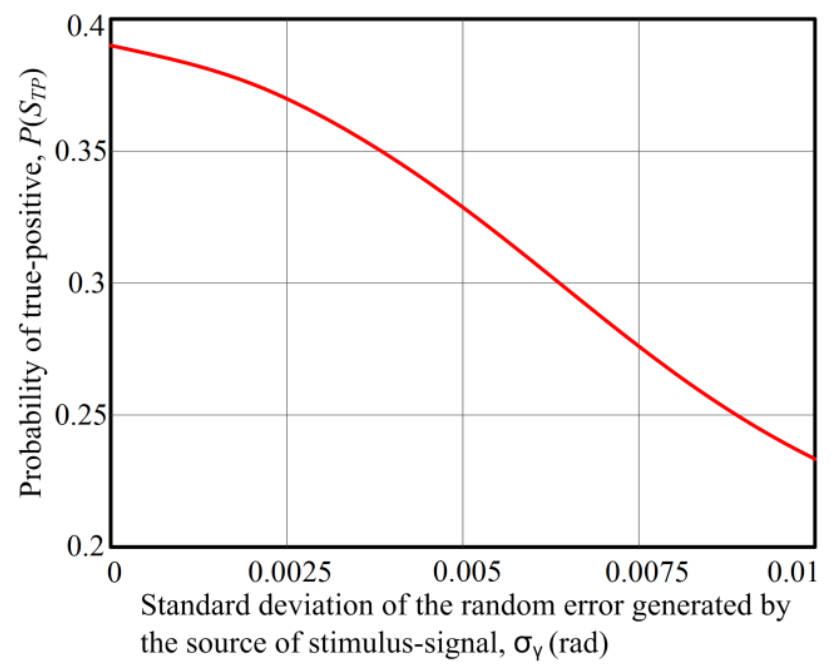

Figure 8 . Dependence of the probability of true-positive versus standard deviation of the random error generated by the source of the stimulus signal

Figures 9 and 10 show the dependence of the probabilities of false-negative and true-negative versus standard deviation of the random error generated by the SSS.

From Figures 9 and 10, we can conclude that the probability of false-negative increases and the probability of truenegative decreases with a rise in the standard deviation $\sigma_{\gamma}$ from 0 to $0.01 \mathrm{rad}$. However, the impact of the standard deviation of the random error generated by the SSS on probabilities $P\left(S_{F N}\right)$ and $P\left(S_{T N}\right)$ is less than on probabilities $P\left(S_{F P}\right)$ and $P\left(S_{T P}\right)$. Indeed, when $\sigma_{\gamma}$ changes from 0 to 0.01 rad, the probability $P\left(S_{F N}\right)$ increases from $2 \%$ to $7.5 \%$, and the probability $P\left(S_{T N}\right)$ decreases from $57.8 \%$ to $53 \%$. 


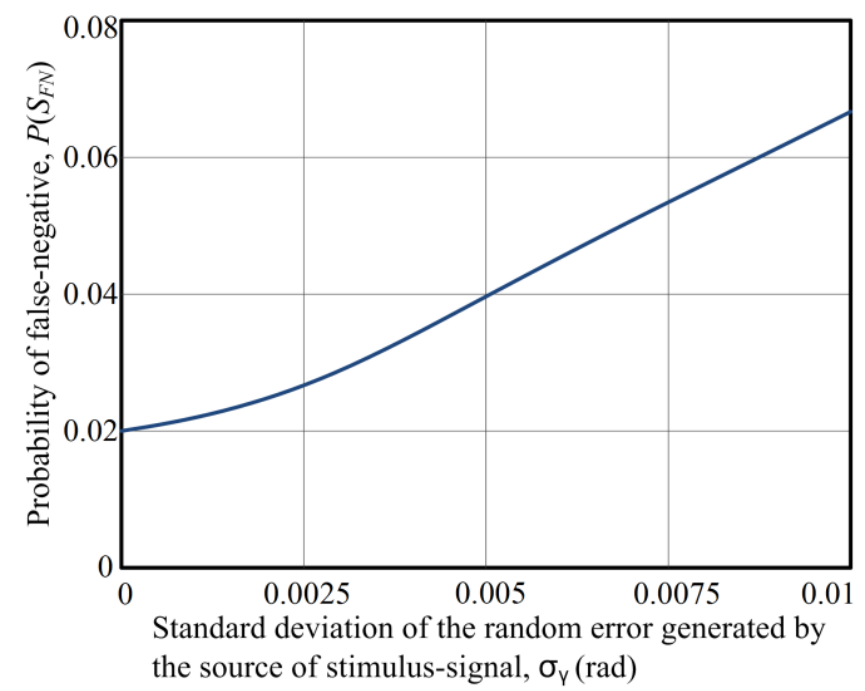

Figure 9. Dependence of the probability of false-negative versus standard deviation of the random error generated by the source of the stimulus signal

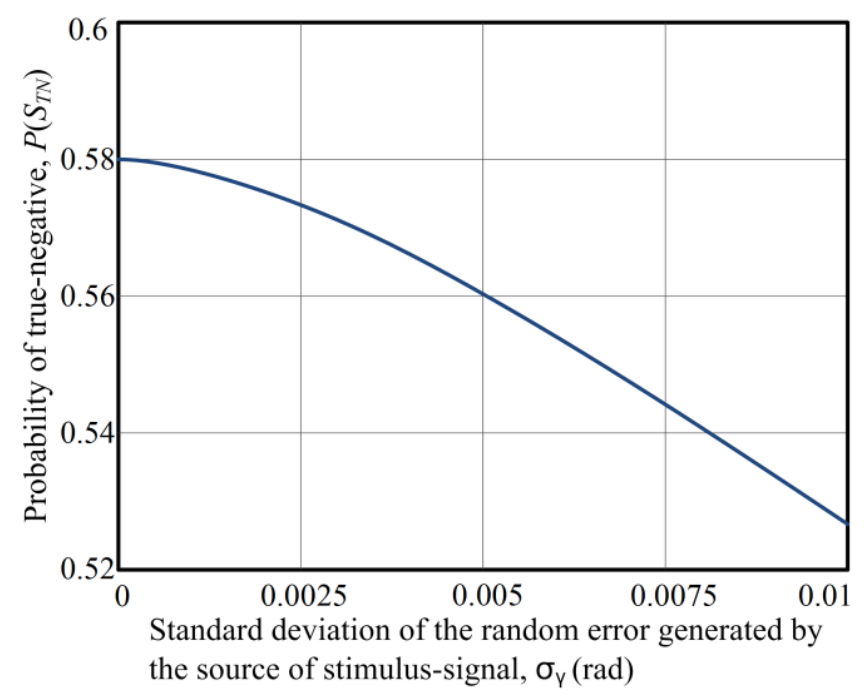

Figure 10. Dependence of the probability of true-negative versus standard deviation of the random error generated by the source of the stimulus signal

Figures 11-14 show the dependences of the probabilities of false-positive, true-positive, false-negative, and true-negative on the standard deviation of the random component of the azimuth measurement error $\sigma_{\xi}$ when $\sigma_{\gamma}=0$. Analyzing Figures 11-14, we can make the following conclusions: 1) even with a zero random component of the SSS error $(\Gamma=0)$, the probabilities of $P\left(S_{F P}\right)$ and $P\left(S_{F N}\right)$ are different from zero due to the presence of a random component of the azimuth measurement error $\Xi$ created by the VOR receiver, 2) the dependence of the trustworthiness indicators on the standard deviation of the random component of the azimuth measurement error $\sigma_{\xi}$ is similar to that of $\sigma_{\gamma}$. However, the dependence of the probabilities $P\left(S_{F N}\right)$ and $P\left(S_{T N}\right)$ on the standard deviation $\sigma_{\xi}$ is linear when $\sigma_{\gamma}=0$. Moreover, the indicators reach their extreme values, $P\left(S_{F P}\right)=P\left(S_{F N}\right)=0$, $P\left(S_{T P}\right)=\lambda_{0} / \Lambda$, and $P\left(S_{T N}\right)=\left(\lambda_{1}+\lambda_{2}\right) / \Lambda$ at $\sigma_{\xi}=0$.

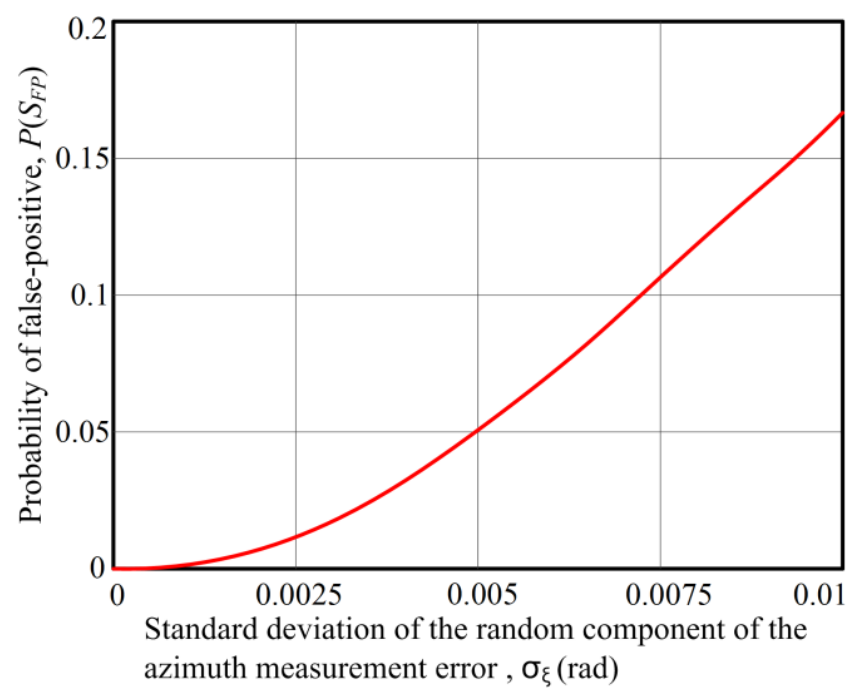

Figure 11. Dependence of the probability of false-positive versus standard deviation of the random component of the azimuth measurement error

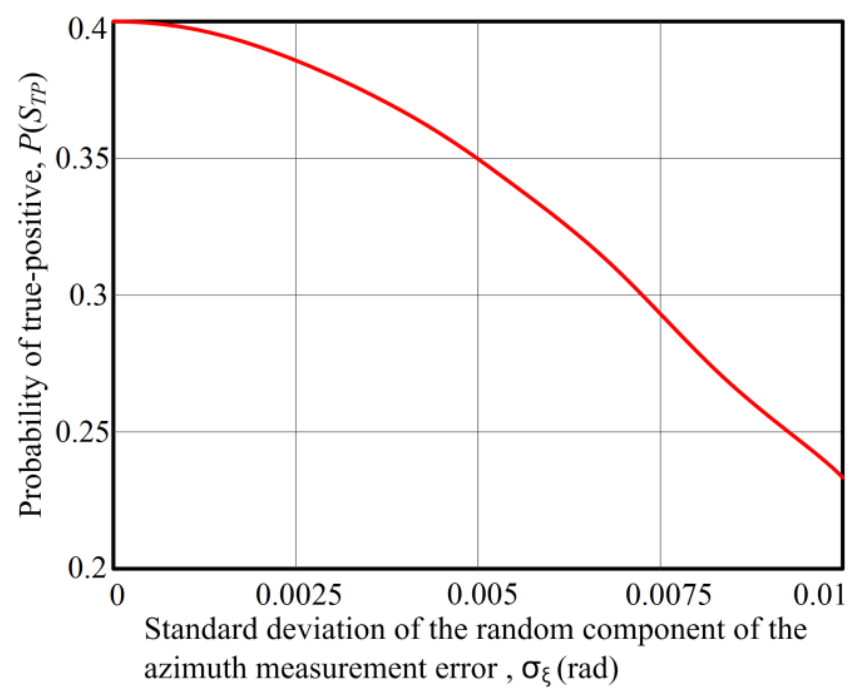

Figure 12. Dependence of the probability of true-positive versus standard deviation of the random component of the azimuth measurement error

Example 2. Let us consider an example of calculating the trustworthiness indicators when monitoring the health of dismounted LRU, which is an onboard ILS designed to land aircraft using ILS beacons of the meter range. Two main parameters of the dismounted LRU are tested: course channel centering error and glissade channel centering error. We use the following initial data. For course channel centering error: $|\Delta|=0.0077 \mathrm{DDM}$ (the difference in the depth of modulation), $\Delta_{h}=-\Delta_{l}=0.155 \mathrm{DDM}, \sigma_{\theta}=0.0025 \mathrm{DDM}, \sigma_{\xi}$ $=0.0023 \mathrm{DDM}$, and $\sigma_{\gamma}=0.0015 \mathrm{DDM}$. For glissade channel centering error: $|\Delta|=0.014 \mathrm{DDM}, \Delta_{h}=-\Delta_{l}=0.175 \mathrm{DDM}$, 
$\sigma_{\theta}=0.00469 \mathrm{DDM}, \quad \sigma_{\xi}=0.0042 \mathrm{DDM}, \quad$ and $\quad \sigma_{\gamma}=$ 0.0017 DDM. As in the case of Example 1, we assume that $\lambda_{0} / \Lambda=0.4$ and $\lambda_{1} / \Lambda=\lambda_{2} / \Lambda=0.3$.

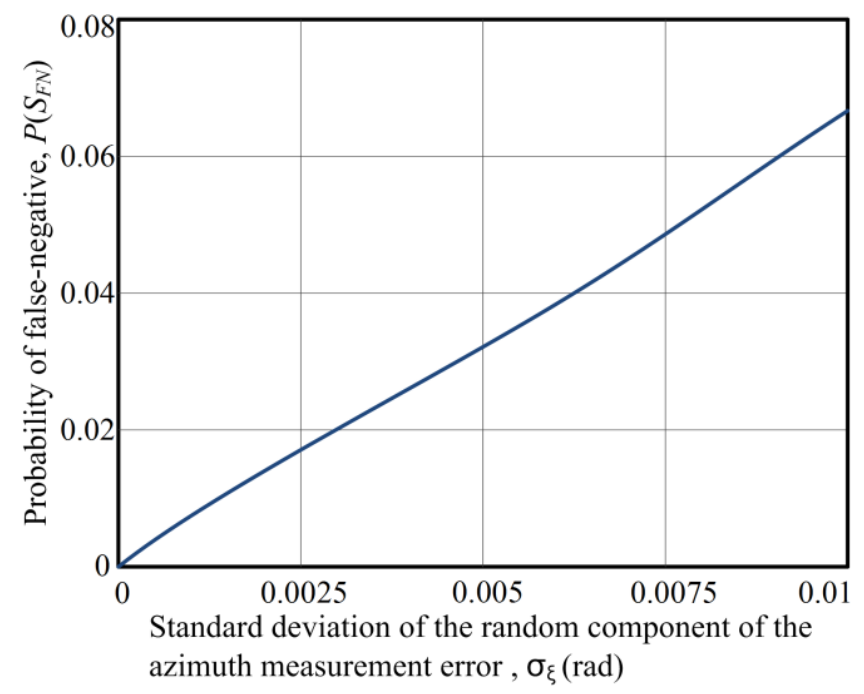

Figure 13. Dependence of the probability of false-negative versus standard deviation of the random component of the azimuth measurement error

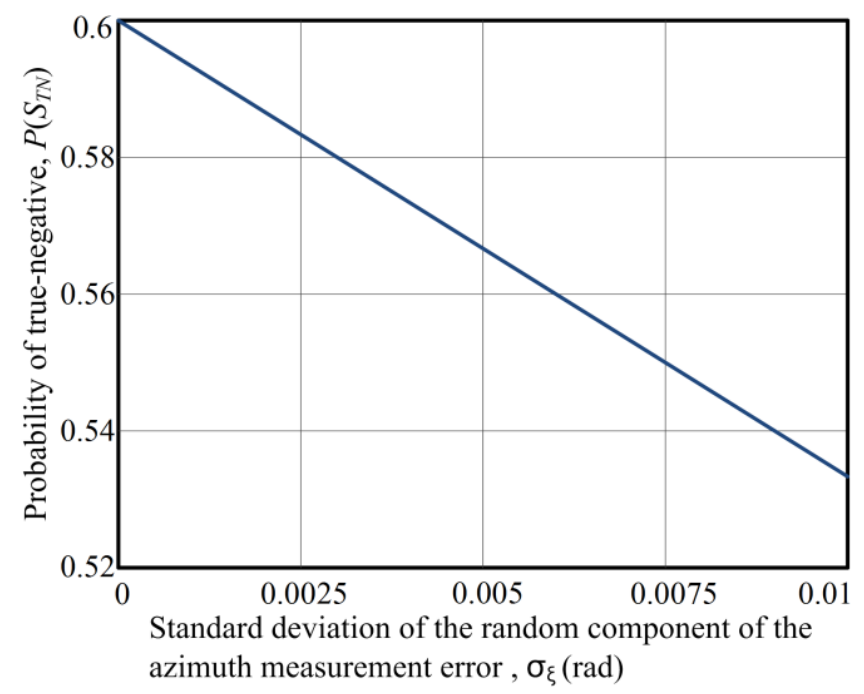

Figure 14. Dependence of the probability of true-negative versus standard deviation of the random component of the azimuth measurement error

Table 2 shows the calculated values of the trustworthiness indicators. As we can see in Table 2, the trustworthiness of health monitoring ILS using ATE is relatively high. The probability of a false-positive and a false-negative does not exceed $1.5 \%$ and $0.67 \%$, respectively.

The probability of correct testing of the ILS receiver by two parameters, we calculate by the formula of Kudritsky et al. (1977):

$$
D=\prod_{i=1}^{2}\left[1-P\left(S_{F P}\right)_{i}-P\left(S_{F N}\right)_{i}\right]=0.962
$$

where $P\left(S_{F P}\right)_{i}$ and $P\left(S_{F N}\right)_{i}$ are, respectively, the probabilities of false-positive and false-negative when testing $i$-th parameter.

Table 2. Calculated trustworthiness indicators of tested ILS LRU

\begin{tabular}{l|c|c}
\hline \multirow{2}{*}{$\begin{array}{l}\text { Trust- } \\
\text { worthiness } \\
\text { indicator }\end{array}$} & \multicolumn{2}{|c}{ Tested parameter } \\
\cline { 2 - 3 } & $\begin{array}{c}\text { Course channel } \\
\text { centering error }\end{array}$ & $\begin{array}{c}\text { Glissade channel } \\
\text { centering error }\end{array}$ \\
\hline $\begin{array}{l}\text { Probability of } \\
\text { false-positive }\end{array}$ & $1.5 \times 10^{-2}$ & $1.2 \times 10^{-2}$ \\
\hline $\begin{array}{l}\text { Probability of } \\
\text { false-negative }\end{array}$ & $4.5 \times 10^{-3}$ & $6.7 \times 10^{-3}$ \\
\hline $\begin{array}{l}\text { Probability of } \\
\text { true-positive }\end{array}$ & 0.3850 & 0.3880 \\
\hline $\begin{array}{l}\text { Probability of } \\
\text { true-negative }\end{array}$ & 0.5955 & 0.5933 \\
\hline
\end{tabular}

\section{Conclusion}

This paper has developed a new mathematical model for assessing the trustworthiness indicators of health monitoring the dismounted avionics systems, which include the probabilities of false-positive, false-negative, true-positive, and true-negative. Using the designed block diagram of the VOR receiver health monitoring, we have formulated the corresponding decision rule. Based on the decision rule, we have derived general equations for computing the probabilities of correct and incorrect decisions when monitoring the health of dismounted avionics systems by ATE. The proposed equations are applicable at arbitrary distributions of monitoring parameters and measurement errors.

We specifically considered the case of an exponential distribution of permanent failures and intermittent faults; then, we derived formulas for calculating the trustworthiness indicators since usually there is statistical information for this distribution. By numerical calculations, we have shown that the probability of false-positive increases, and the probability of true-positive decreases when the standard deviation of the stimulus signal increases. Besides that, the probability of false-positive has a much stronger dependence than that of true-positive. Indeed, the first one rises from $2 \%$ to $17 \%$ when the standard deviation increases from 0 to $0.01 \mathrm{rad}$, whereas the second one decreases from $38 \%$ to $23 \%$. We 
have shown that the probability of false-negative increases and the probability of true-negative decreases with a rise in the standard deviation of the stimulus signal. However, this dependence is not as strict as for probabilities of falsepositive and true-positive. We also demonstrated that even with a zero error generated by the source of the stimulus signal, the probabilities of false-positive and false-negative are different from zero; this is due to the presence of a random component of the azimuth measurement error created by the VOR receiver. Numerical calculations have shown that the trustworthiness of health monitoring the ILS LRU is high enough because a false-positive and a false-negative probability does not exceed $1.5 \%$ and $0.67 \%$, respectively.

Our future work will include developing effectiveness criteria of health monitoring the dismounted avionics systems that affect flight safety or flight regularity. We also plan to elaborate methods for increasing the trustworthiness of health monitoring through repeated measurements and control tolerances.

\section{NOMENCLATURE}

$A$
$\Gamma$
$\Theta$
$\Xi$
$Y$
$\Delta$

$y$
$S_{F P}$
$S_{F N}$
$S_{T P}$
$S_{T N}$
$Z$

$g(z) \quad$ probability density function of random variable $Z$

$f(\xi) \quad$ probability density function of random variable $\Xi$

$\varphi(\gamma) \quad$ probability density function of random variable $\Gamma$

$q(\theta) \quad$ probability density function of random variable $\Theta$

$P\left(S_{F P}\right) \quad$ probability of false-positive

$P\left(S_{F N}\right) \quad$ probability of false-negative

$P\left(S_{T P}\right) \quad$ probability of true-positive

$P\left(S_{T N}\right) \quad$ probability of true-negative

$\Theta_{0} \quad$ systematic error of azimuth measurement

$\Theta_{1} \quad$ systematic error of azimuth measurement when a permanent failure occurs, resulting in a reduction of systematic error below the permissible limit

$\Theta_{2} \quad$ systematic error of azimuth measurement when a permanent failure occurs, resulting in an increase of systematic error over the permissible limit

$\lambda_{0} \quad$ rate of intermittent faults transferring the VOR receiver to the state in which it is dismounted from the aircraft board, not having any permanent failure

$\lambda_{1} \quad$ rate of permanent failures that transfer VOR receiver from the operable state to inoperable state corresponding to a decrease in systematic error by an amount higher than permissible

$\lambda_{2}$ rate of permanent failures that transfer VOR receiver from the operable state to inoperable state corresponding to an increase in systematic error by an amount higher than permissible

$\Lambda \quad$ total transition rate

$q(\theta) \quad$ probability density function of systematic component of the azimuth measurement error over the set of the same VOR receivers

$q_{0}(\theta) \quad$ probability density function of systematic component of the azimuth measurement error in the absence of permanent failures

$q_{1}(\theta) \quad$ probability density function of systematic component of the azimuth measurement error when a permanent failure occurs, resulting in a reduction of systematic error below the permissible limit

$q_{2}(\theta) \quad$ probability density function of systematic component of the azimuth measurement error when a permanent failure occurs, resulting in an increase of systematic error over the permissible limit

$\sigma_{\xi} \quad$ standard deviation of random variable $\Xi$

$\sigma_{\gamma} \quad$ standard deviation of random variable $\Gamma$

$\sigma_{\theta} \quad$ standard deviation of random variable $\Theta$

$\mathrm{D}_{0} \quad$ state of VOR receiver in which it is dismounted from the aircraft board not having any permanent failure

$\mathrm{D}_{1} \quad$ state of VOR receiver in which it is dismounted from the aircraft board due to a permanent failure corresponding to a decrease in systematic error by an amount higher than permissible

$\mathrm{D}_{2} \quad$ state of VOR receiver in which it is dismounted from the aircraft board due to a permanent failure corresponding to an increase in systematic error by an amount higher than permissible

$\Delta_{h} \quad$ higher boundary of the systematic component of the error in azimuth measurement in the presence of defects in the VOR receiver

$\Delta_{l} \quad$ lower boundary of the systematic component of the error in azimuth measurement in the presence of defects in the VOR receiver normalization constant 


\section{AbBreviations}

$\begin{array}{ll}\text { ATE } & \text { Automated test equipment } \\ \text { BITE } & \text { Built-in test equipment } \\ \text { D-level } & \text { Depot maintenance } \\ \text { eCASS } & \begin{array}{l}\text { electronic Consolidated Automated } \\ \text { Support System }\end{array} \\ \text { IEC } & \text { International Electrotechnical } \\ & \text { Commission } \\ \text { I-level } & \text { Intermediate maintenance } \\ \text { ILS } & \text { Instrument landing system } \\ \text { LRM } & \text { Line-replaceable module } \\ \text { LRU } & \text { Line-replaceable unit } \\ \text { O-level } & \text { Organizational maintenance } \\ \text { PDF } & \text { Probability density function } \\ \text { PXI } & \text { Peripheral component interconnect } \\ & \text { extension for instrumentation } \\ \text { SRU } & \text { Shop replaceable unit } \\ \text { SSS } & \text { Source of stimulus signal } \\ \text { VOR } & \text { Very High-Frequency Omni- } \\ & \text { Directional Range }\end{array}$

\section{REFERENCES}

Aeronautical Information Manual (2017). Official Guide to Basic Flight Information and ATC Procedures, U.S. Department of Transportation. Federal Aviation Administration, USA. http://www.faa.gov/atpubs

Aeroflex (2005). Avionics IRIS $® 2000$ automatic test equipment system. Plainview, New York, USA. https://www.avionteq.com/document/IRIS-2000Specification-Sheet.pdf

Anderson, K. (2014). Intermittent fault detection \& isolation system (IFDIS). Aerospace \& defense. Roy, USA. https://contest.techbriefs.com/2014/entries/aerospaceand-defense $/ 4014$

Bao, H., Ying, C. L., Shi, Q. W., \& Teng, J. F. (2006). Research on portable maintenance aid equipment using ARM-based VXI modules. Proceedings of Sixth International Symposium on Instrumentation and Control Technology. October 13-15, Beijing, China. doi: https://doi.org/10.1117/12.718027

Breitgand, D., Goldstein, M., Henis, E., \& Shehory, O. (2011). Efficient control of false negative and false positive errors with separate adaptive thresholds. IEEE Trans. on Network and Service Management, 8(2), 128140. doi: 10.1109/TNSM.2011.020111.00055

Chen, X., Zhang, Z., \& Zhang, Z. (2019). Real-time equipment condition assessment for a class-imbalanced dataset based on heterogeneous ensemble learning. Eksploatacja $i$ Niezawodnosc - Maintenance and Reliability, 21(1), 68-80. doi: http://dx.doi.org/10.17531/ein.2019.1.9

Droste, D. B., \& Guilbeaux, G. (2009). Advanced architecture for achieving true vertical testability in next generation ATE. Proceedings of IEEE Autotestcon. 1417 September, Anaheim, California. doi: 10.1109/AUTEST.2009.5314088

Ebrahimi, N. (2008). Simultaneous control of false positives and false negatives in multiple hypotheses testing. Journal of Multivariate Analysis, 99(3), 437-450. doi: https://doi.org/10.1016/j.jmva.2006.12.006

eCASS electronic consolidated automated support system (2020). Lockheed Martin. Bethesda, Maryland, USA. https://www.lockheedmartin.com/en-us/products/ electronic-consolidated-automated-support-systemecass.html.

Evlanov, L. G. (1979). Control of dynamic systems. Moscow: Nauka.

Foss, A., \& Zaiane, O. R. (2008). Estimating true and false positive rates in higher dimensional problems and its data mining applications. Proceedings of IEEE International Conference on Data Mining Workshops. 15-19 December, Pisa, Italy. doi: 10.1109/ICDMW.2008.38

Hand, D., \& Christen, P. (2018). A note on using the Fmeasure for evaluating record linkage algorithms. Statistics and Computing, 28(3), 539-547. doi: https://doi.org/10.1007/s11222-017-9746-6

Ho, C. Y., Lai, Y. C., Chen, I. W, Wang, F. Y. \& Tai, W. H. (2012). Statistical analysis of false positives and false negatives from real traffic with intrusion detection/prevention systems. IEEE Communications Magazine, 50(3), 146-154. doi: 10.1109/MCOM.2012.6163595

Hossin, M., \& Sulaiman, M. N. (2015). A review on evaluation metrics for data classification evaluations. Int. J. of Data Mining \& Knowledge Management Process, 5(2), 1-11. doi: 10.5121/ijdkp.2015.5201

International Electrotechnical Commission (IEC) (2004). Higher performance protocol for the standard digital interface for programmable instrumentation - Part 1: General. In IEC, IEC-60488-1: 2004. Part 1 (p. 13). Genève, Switzerland: International Electrotechnical Commission.

International Electrotechnical Commission (IEC) (2004). Standard Digital Interface for Programmable Instrumentation - Part 2: Codes, formats, protocols and common commands. In IEC, IEC-60488-2: 2004. Part 2. Genève, Switzerland: International Electrotechnical Commission.

Ilarslan, M., \& Ungar, L. Y. (2016). Mitigating the impact of false alarms and no fault found events in military systems. IEEE Instrumentation \& Measurement Magazine, 8, 16-22. doi: 10.1109/MIM.2016.7524202

Khan, S, Phillips, P., Hockley, C. \& Jennions, I. (2014). No fault found events in maintenance engineering part 2: Root causes, technical developments and future research. Reliability Eng. \& System Safety, 123, 196208. doi: https://doi.org/10.1016/j.ress.2013.10.013 
Kudritsky, V. D., Sinitsa, M. A., \& Chinaev, P. I. (1977). Automation of health-monitoring of electronic equipment. Moscow: Soviet Radio.

Lisman, J. H. C., \& van Zuylen, M. C. A. (1972). Note on the generation of most probable frequency distributions. Statistica Neerlandica, 26(1), 19-23. doi: https://doi.org/10.1111/j.1467-9574.1972.tb00152.x

Ma, L., Zhang, X., Wang, K. \& Xu, T. (2013). A general method for module automatic testing in avionics systems. Research Journal of Applied Sciences, Engineering and Technology, 5(20), 4796-4804. doi: 10.19026/rjaset.5.4322

Malesich, M. (2007). Advances in DoD'S ATS framework. Proceedings of IEEE Autotestcon. 17-20 September, Baltimore, MD, USA. doi: 10.1109/AUTEST.2007.4374202

Mane, S., Srivastava, J., Hwang, S. Y., \& Vayghan, J. (2004). Estimation of false negatives in classification. Proceedings of Fourth IEEE International Conference on Data Mining. 1-4 November, Brighton, UK. doi: 10.1109/ICDM.2004.10048

Manning, C. D., Raghavan, P., \& Schutze, H. (2008). Introduction to information retrieval. Cambridge: Cambridge University Press.

Rausand, M., \& Hoyland, A. (2003). System reliability theory: models, statistical methods, and applications. New York: Wiley.

Raza, A. (2018). Maintenance model of digital avionics. Aerospace, 5(2), 1-16. doi: https://doi.org/10.3390/aerospace5020038

Raza, A., Ulanskyi, V., Augustynek, K., \& Warwas, K. (2017). Generalized cost functions of avionics breakdown maintenance strategy. Proceedings of IEEE Aerospace Conference. 4-11 March, Big Sky, MT. doi: 10.1109/AERO.2017.7943754

Raza, A., \& Ulansky, V. (2018). Modelling of false alarms and intermittent faults and their impact on the maintenance cost of digital avionics. Procedia Manufacturing, 16, 107-114. doi: https://doi.org/10.1016/j.promfg.2018.10.171

Raza, A., \& Ulansky, V. (2018). Analyses of warranty losses to avionics suppliers. Proceedings of IEEE Aerospace Conference. 3-10 March, Big Sky, MT. doi: 10.1109/AERO.2018.8396752

Raza, A., \& Ulansky, V. (2021). Through-life maintenance cost of digital avionics. Applied Sciences, 11(2), 1-30. doi: https://doi.org/10.3390/app11020715

Ross, W. A. (2003). The impact of next generation test technology on aviation maintenance. Proceedings of IEEE Autotestcon. 23-25 September, Anaheim, CA, USA. doi: 10.1109/AUTEST.2003.1243547

Scott, C. (2007). Performance measures for NeymanPearson classification. IEEE Trans. on Information Theory, 53(8), 2852-2863. doi: 10.1109/TIT.2007.901152
Sokolova, M., Japkowicz, N., \& Szpakowicz S. (2006). Beyond accuracy, F-score and ROC: a family of discriminant measures for performance evaluation. Proceedings of 19th Australian Joint Conference on Artificial Intelligence: Advances in Artificial Intelligence. 4-8 December, Hobart, TAS, Australia. doi: https://doi.org/10.1007/11941439_114

Stora, M. J., \& Droste D. (2003). ATE open system platform. IEEE-P1552 structured architecture for test systems (SATS). Proceedings of IEEE Autotestcon. 22-25 September, Anaheim, CA, USA. doi: 10.1109/AUTEST.2003.1243559

Spherea (2017). The prepared maintenance solution for airlines and MROS. Hamburg, Germany. https:// www.spherea.de/en/atec-series/

Ulansky, V. (1992). The trustworthiness of multiplemonitoring the operability of nonrepairable electronic systems. In Ignatov V. (Ed.), Saving Technologies and Avionics Maintenance of Civil Aviation Aircraft (1425). Kyiv: KIIGA Press.

\section{BIOGRAPHIES}

Vladimir Ulansky received an M.Sc. degree in aeronautical radio engineering from the National Aviation University (Kyiv, Ukraine) in 1975. He received a $\mathrm{Ph} . \mathrm{D}$. degree in technical cybernetics in 1981 from the National Technical University of Ukraine "Igor Sikorsky Kyiv Polytechnic Institute" and D.Sc. degree in aeronautical engineering from National Aviation University in 1989. He received Best Presented Paper Award in the 4th International Conference on Through-life Engineering Services (Cranfield, UK) in 2015. He became a Fellow of the Institute of Mathematics and its Applications (UK) in 2017 and a Senior Member of the Institute of Electrical and Electronics Engineers (US) in 2020. He is currently a Professor in the Department of Electronics, Robotics, Monitoring and IoT Technologies, National Aviation University. His current research interests include RF electronics, avionics maintenance, conditionbased and predictive maintenance.

Igor Machalin received the M.Sc. and Ph.D. degrees in aeronautical radio engineering from the National Aviation University (Kyiv, Ukraine) in 1980 and 1988. He received a D.Sc. degree in aeronautical engineering from National Aviation University in 2010. He is currently a Professor in the Department of Telecommunication and Radio Electronic Systems, National Aviation University. His current research interests include operation, maintenance, and design of aeronautical radio engineering and telecommunication systems.

Iryna Terentyeva received an M.Sc. in radio engineering from the National Aviation University (Kyiv, Ukraine) in 1999. She received a Ph.D. degree in radio engineering and television systems from National Aviation University in 2018. She is currently an Associate Professor in the 
Department of Telecommunication and Radio Electronic Systems, National Aviation University. Her current research interests include mathematical modeling of operational reliability of communication systems. 